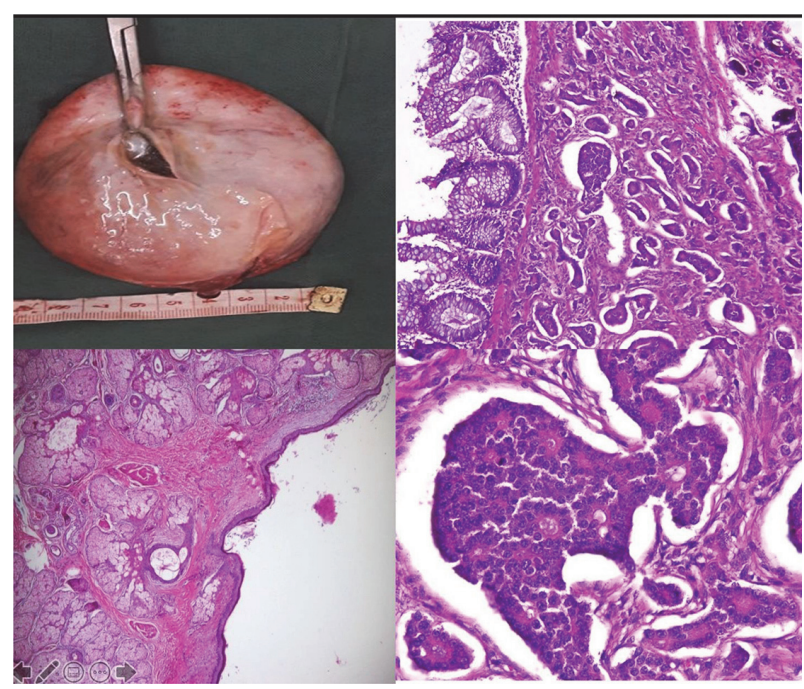

Abstract 424 Figure 1

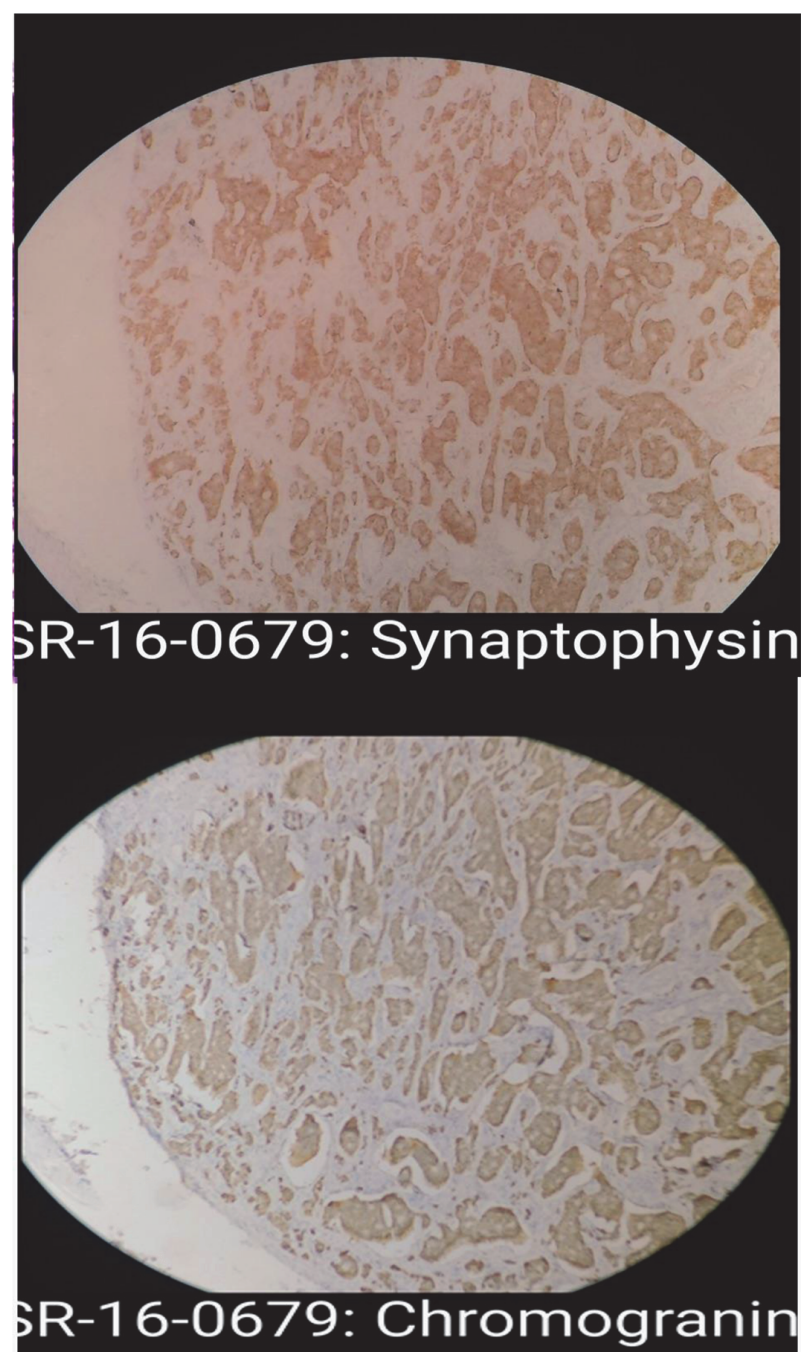

Abstract 424 Figure 2

carcinoid tumor is surgical excision regardless of histologic type. Thorough histopathologic examination and extensive sampling of a dermoid cyst is necessary to detect malignant transformation.
IGCS20_1462

\section{PATTERNS OF RECURRENCE IN LOW-RISK ENDOMETRIAL CANCER- EVIDENCE FOR A CHANGE IN FOLLOW-UP}

Y Naaman*, T Hodge, A Jones, F Chin, D Neesham, O McNally. Gynaecology-Oncology unit, The Royal Women's Hospital, Australia

\subsection{6/ijgc-2020-IGCS.369}

Introduction The recurrence rate of low-risk endometrial cancer is reported to be very low. Given the high prevalence of the disease and the low risk of recurrence in this population, the yield from routine gynecological oncology follow up for this group of women is questionable.

Objective To describe the rate and patterns of recurrence in low-risk endometrial cancer in patients with low-risk endometrial cancer.

Methods A retrospective study of all patients with stage 1A, grade 1-2 endometrial cancer that had primary surgical treatment and follow up in our centre was conducted. For patients with recurrent disease, demographics, site of recurrence, presentation, salvage treatment and long-term outcomes were analyzed.

Results 1215 Low-risk endometrial cancer patients were treated with primary surgery between 1981 and 2018. Of these, 24 patients were identified as having had recurrent disease $(1.97 \%)$. In most patient's recurrent disease $(17 / 24$ - 70\%) was at the vault/locoregional and was deemed salvageable. Median time to recurrence was three years (range 1-8 years), and 12 patients (50\%) were asymptomatic. Of those with symptoms, the most common presenting symptoms of recurrence were vaginal bleeding and abdominal pain.

Conclusion The incidence of recurrent disease in women with low-risk endometrial cancer is low, less than $2 \%$, and indeed is lower than the risk for endometrial cancer in the general population. Hence, the value of routine tertiary follow up is questionable, and alternative models, including communitybased review and patient report symptoms, should be strongly supported.

\section{IGCS20_1463}

\section{THE DIAGNOSTIC VALUE OF MICRORNA SIGNATURE IN ENDOMETRIAL CANCER}

${ }^{1} \mathrm{H}$ Donkers ${ }^{*},{ }^{2} \mathrm{M}$ Hirschfeld, ${ }^{2} \mathrm{D}$ Weiß, ${ }^{2} \mathrm{~T}$ Erbes, ${ }^{2} \mathrm{M}$ Jaeger, ${ }^{3} \mathrm{~J}$ Pijnenborg, ${ }^{4} \mathrm{R}$ Bekkers, ${ }^{1} \mathrm{~K}$ Galaal. ${ }^{1}$ Royal Cornwall Hospital, UK; ${ }^{2}$ University of Freiburg, Germany; ${ }^{3}$ Radboud University Medical Centre, Netherlands; ${ }^{4}$ Maastricht University, Netherlands

\subsection{6/ijgc-2020-IGCS.370}

Background MiRNAs are noncoding RNAs that regulate gene expression and contribute to the development of cancer. MiRNAs have been shown to be stable in urine, serum and tissue samples. They may be promising biomarkers for non-invasive detection of EC.

Methods A retrospective cohort study of women diagnosed with EC between January 2017 and December 2017 was performed at the Royal Cornwall Hospital. Archived formalinfixed paraffin-embedded (FFPE) samples were obtained from 